\title{
NEONATAL BLOODSTREAM INFECTION SURVEILLANCE IN THE UK USING NATIONALLY AGREED DEFINITIONS
}

\author{
A.K. Mahaveer, A. Richardson, T. Neal, N. Subhedar \\ Liverpool Women's Hospital, Liverpool, UK
}

Background and aims: Various definitions of bloodstream infection (BSI) and catheter-associated BSI (CABSI) are used internationally. Until recently there have been no nationally accepted definitions in the UK. We aimed to collect data on BSI and CABSI using these new standardised definitions.

Methods: Information about all late-onset bacteraemias in preterm infants $<30$ weeks' gestation and/or $<$ $1500 \mathrm{~g}$ was collected prospectively over a 12 month period from a microbiology database based in a level 3 UK NICU. Information about clinical presentation at the time when the infection screen was performed was entered prospectively into an electronic patient data management system. The diagnosis of BSI was made either when the blood culture yielded,

(1) a pure growth of a recognised pathogen;

(2) a skin commensal or mixed growth plus 3 or more clinical signs.

CABSI was diagnosed when a central vascular catheter was in place within 48 hours of the blood culture being taken.

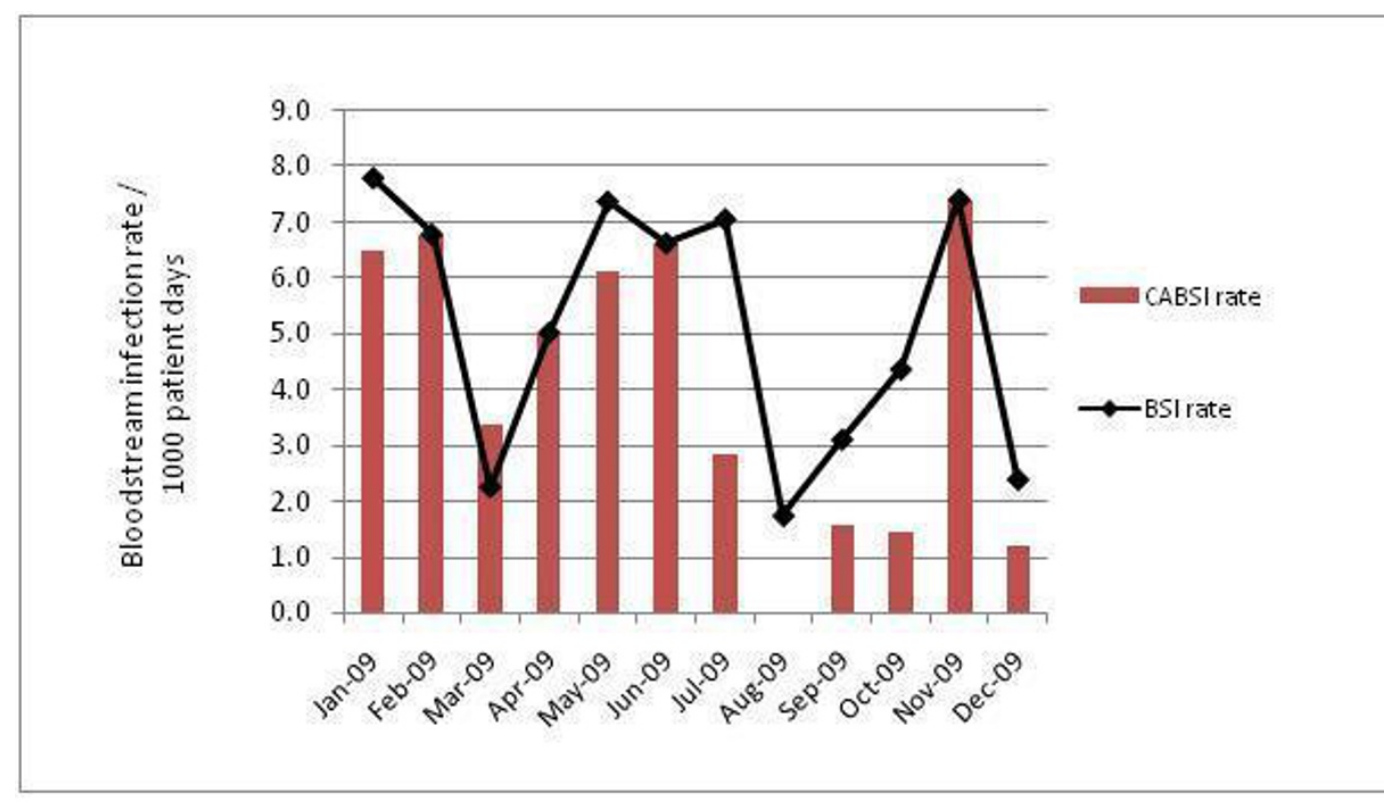

\section{[Graph 1]}

The median (range) rate of BSI and CABSI was 5.8 (1.7-7.8) and $4.2(0-7.4)$ per 1000 patient days, respectively. Approximately three-quarters of all late-onset BSI were CABSIs.

Conclusions: This study demonstrates the feasibility of a system designed to collect BSI data for surveillance purposes using nationally agreed definitions. These definitions will be used to provide information about late-onset BSI for national surveillance in the UK. 